\title{
Metodología para la integración de la gestión en una pyme del sector ferretero en Colombia*
}

\author{
Methodology for management integration in a medium-sized \\ enterprise in the hardware sector in Colombia
}

\section{Metodologia para a integração da gestão em uma PME do setor de ferragens na Colômbia}

Erika Rocio Rodríguez Rodrígue****

Universidad Santo Tomás, Colombia

Carlos Enrique Montenegro Narváä*****

Universidad Santo Tomás, Colombia

Cómo citar: Rodríguez Rodríguez, E. R. y Montenegro Narváez, C. E. (2019). Metodología para la integración de la gestión en una pyme del sector ferretero en Colombia. Signos. Investigación en Sistemas de Gestión, 11(2), 83-98. Doi: https://doi.org/10.15332/24631140.5083

\section{RESUMEN}

En Colombia los empresarios mipyme buscan herramientas que contribuyan a la perdurabilidad y competitividad de sus negocios, mediante la superación de los problemas más comunes de dichas empresas: ciclos de vida cortos y bajo crecimiento. Dado que estas organizaciones representan más del $90 \%$ del sector productivo nacional y son responsables del $35 \%$ del PIB de acuerdo con lo reportado por el Dane, es importante para el país que sean competitivas y sostenibles. Es por ello que se desarrolló una metodología para la integración de la

* Artículo de investigación.

** Magíster en Calidad y Gestión Integral, administradora de empresas. Bogotá, Colombia. Correo electrónico: erika.rodriguezr@usantotomas.edu.co. Orcid: https://orcid.org/0000-0002-6445-324X

*** Magíster en calidad y Gestión Integral, especialista en Gerencia de Proyectos en Ingeniería de Telecomunicaciones, ingeniero electrónico. Decano Facultad de Ingeniería Electrónica, Universidad Santo Tomás, Bogotá, Colombia. Correo electrónico: dec.ingelectronica@usantotomas.edu.co. Orcid: https://orcid.org/0000-0002-6445-324X 
gestión de estas empresas. La investigación siguió un diseño explicativo secuencial y la metodología resultante fue sometida a un panel de expertos que tuvo en cuenta los criterios de pertinencia y relevancia. Asimismo, se consideró el método estadístico para validar el contenido a partir de las ecuaciones de Lawshe con el factor de corrección propuesto por Tristán-López. Los resultados evidencian que la metodología propuesta permite la integración de la gestión, al obtener resultados en cuanto a validez de contenido en el criterio de pertinencia (IVC $=0.8542)$, el de relevancia $(I V C=0.9167)$ y el alfa de Cronbach $(\alpha=0.9886)$. Se concluye que la metodología diseñada provee los insumos necesarios para integrar la gestión a partir de la dirección estratégica y es aplicable al escenario estudiado.

Palabras clave: calidad, ISO 9001, seguridad y salud en el trabajo, sistemas de gestión integrados.

\section{ABSTRACT}

In Colombia, micro, small and medium-sized enterprises are searching for tools contributing to perdurability and competitiveness of their businesses, overcoming the most common problems of micro, small and medium-sized enterprises: short lifecycles and low growth. Since these organizations account for over $90 \%$ of the domestic production sector and are responsible for $35 \%$ of GDP as reported by DANE, it is important to the country that these organizations be competitive and sustainable. As a consequence of this, a methodology was developed for management integration for these enterprises. The research was developed following a sequential explanatory design, and the methodology designed was submitted to an expert panel taking into account pertinence and relevance criteria. Likewise, the statistical method was considered for content validity based on Lawshe formula with the correction factor proposed by
Tristán-López. According to the results, the proposed methodology makes it possible for management to be integrated, as shown by the results of the content validity criteria, such as pertinence $(\mathrm{IVC}=0.8542)$, relevance $(\mathrm{IVC}=0.9167)$ and Cronbach's alpha $(\alpha=0.9886)$. It is concluded that the methodology designed provides the input required for management integration based on the strategic direction and is applicable to the studio scenario.

Keywords: Quality, ISO 9001, occupational safety and health, integrated management systems.

\section{RESUMO}

$\mathrm{Na}$ Colombia, os empresários MPE procuram ferramentas que contribuam a longevidade e competitividade dos seus negócios, superando os problemas mais comuns das PMEs: ciclos de vida curtos e de baixo crescimento. Como essas organizações representam mais de $90 \%$ do setor produtivo nacional e são responsáveis de $35 \%$ do PIB conforme o relatado pelo DANE, é importante para o país que elas sejam competitivas e sustentáveis. É por isso que foi desenvolvida uma metodologia para a integração da gestão voltada a essas empresas. A pesquisa foi desenvolvida conforme um design explicativo sequencial e a metodologia projetada foi submetida a um painel de especialistas levando em conta os critérios de pertinência e relevância. Igualmente, foi considerado o método estatístico para a validade do conteúdo partindo das equações de Lawshe com o fator de correção colocado por Tristán-López. Os resultados revelam que a metodologia colocada permite a integração da gestão, obtendo resultados nos critérios de validade de conteúdo no critério de pertinência $(\mathrm{IVC}=0,8542$ ), relevância $(\mathrm{IVC}=0,9167)$ e um coeficiente alfa de Cronbach $(\alpha=0,9886)$. Conclui-se que a metodologia projetada provê insumos necessários para integrar a 
gestão partindo do direcionamento estratégico e é aplicável ao cenário de estudo.

Palavras-chave: qualidade, ISO 9001, segurança e saúde no trabalho, sistemas de gestão integrados.

\section{INTRODUCCIÓN}

En un mundo cada vez más globalizado, donde las exigencias de los mercados y las regulaciones gubernamentales definen nuevos parámetros para la satisfacción de necesidades y la protección de los consumidores en un marco de sostenibilidad económica, social y ambiental, es necesario establecer en las organizaciones mecanismos de gestión que ofrezcan ventajas en términos de competitividad y sostenibilidad. Ese entorno exige generación de valor a través de una capacidad operativa que permita la satisfacción de las necesidades de las partes interesadas cumpliendo con resultados de rentabilidad.

Según el IPA (indicador pyme Anif) ${ }^{1}$ para el segundo trimestre de 2018 el $47 \%$ de las pequeñas organizaciones encuestadas en Colombia realizaron acciones de mejoramiento con el objetivo de aumentar la productividad. La principal acción en ese sentido fue capacitar al personal (31\%; la segunda, obtener una certificación de calidad (12\%) y la tercera, diversificar o ampliar sus mercados en Colombia (6\%). Por lo anterior, se destaca la importancia que tiene para los empresarios pyme la implementación de sistemas de gestión que contribuyan a una futura certificación. Sin embargo, tal implementación implica destinar recursos como tiempo y dinero, que permitan garantizar la debida planeación de los sistemas y su integración. Asimismo, otras dificultades que presentan

1 Este indicador representa una contribución analítica para resumir en un solo valor el clima en el que se desarrollan los negocios en el segmento pyme de Colombia. las pequeñas organizaciones específicamente del sector comercio son la falta de demanda y la competencia con las grandes superficies, según los resultados presentados durante el segundo semestre de 2018 (Centro de Estudios Económicos, 2018).

Salazar-Castillo y Zarandona-Azkuenaga (2007) indican que los sistemas de gestión son herramientas para la construcción de una ventaja competitiva reduciendo costos y aumentando la productividad. Sin embargo, implican el compromiso de la alta dirección y la definición de procesos. Asimismo, afirman dichos autores, la calidad contribuye a conseguir objetivos empresariales que aseguran la continuidad a largo plazo.

De acuerdo con lo anterior, los sistemas de gestión son una oportunidad. Ahora bien, su concepto ha evolucionado y actualmente se busca la integración de esos sistemas para que las organizaciones extiendan su enfoque (Rodríguez-Rojas y Molano, 2018). Según Jørgensen, Remmen y Mellado (2006) esto fortalece la colaboración con las partes interesadas pertinentes y la implementación logra sistemas más útiles, eficientes y sostenibles.

En este sentido, la integración de sistemas de gestión es entendida como un proceso que debe abordarse desde la planeación y que promueve en las organizaciones el diseño y logro de la estrategia. De allí resulta una serie de beneficios asociados con el cumplimiento de la promesa de valor, las metas y los objetivos de la organización, así como con la satisfacción de las partes interesadas (Peña, 2018).

Vieira-Nunhes, Ferreira-Motta y De Oliveira (2017) revelan varios elementos de integración en las empresas. El estudio, realizado en catorce organizaciones de Brasil, determino seis elementos principales para la integración de los sistemas de gestión ISO 9001, ISO 14001 y OHSAS 18001. Los elementos definidos fueron: responsabilidad de la alta dirección, instrucciones 
de trabajo, control de documentos y registros, comunicación, estructura y rendición de cuentas.

El estudio de caso de la presente investigación se realizó en una organización del sector ferretero en Colombia, que se encuentra distribuido en dos categorías: grandes superficies y comercio minorista. El primer grupo está conformado por empresas multinacionales o nacionales con gran capacidad de inversión y recursos organizacionales (Portafolio, 2016). A su vez, al sector minorista lo componen organizaciones tipo pyme, con capacidad económica y estrategia de mercadeo inferiores, lo que las coloca en una posición vulnerable en cuanto a competitividad, crecimiento y sostenibilidad, factores que pueden ser potencializados a través de los sistemas de gestión (Rebelo, Santos y Silva, 2016).

A partir de una revisión de la literatura sobre los sistemas integrados de gestión y el estado de la mipyme objeto de estudio se realizó la investigación. El objetivo fue crear una propuesta metodológica de sistema de gestión integrado bajo los modelos de la norma técnica ISO 9001:2015 y el SG-SST (Sistema de Gestión de la Seguridad y Salud en el trabajo, según el Decreto 1072 de 2015 del Ministerio de Trabajo de Colombia), que impacte el crecimiento y la sostenibilidad de la empresa Electrocerraduras la 85.

En este sentido, el objetivo de esta investigación fue contribuir al crecimiento y la sostenibilidad de una organización mipyme por medio de la integración de la gestión desde la estrategia, alineada con los mapas estratégicos empresariales. El punto de partida fue el propósito organizacional sustentado en el primer objetivo de la investigación: la generación y validación de una metodología de integración del sistema de gestión de la calidad ISO 9001:2015 y el SG-SST en una ferretería tipo pyme en Bogotá. Los siguientes pasos fueron el diagnóstico de la organización, la generación y validación por simulación de la metodología y el análisis de los resultados de la validación. Se procuró actuar equilibrando el compromiso de las personas, incentivando la toma de conciencia y realizando los controles adecuados para la mejora continua.

\section{METODOLOGÍA}

Por la naturaleza de la organización se decidió realizar un estudio con enfoque pragmático. Este comprende análisis organizacionales que dan solución a una problemática real y permite recolectar datos cualitativos y cuantitativos sometidos a una metainferencia (triangulación) posterior.

\section{Diaǵnóstico de la organización}

Teniendo en cuenta el tamaño, la naturaleza y el tiempo de vida de la organización se diseñó un plan de acción para acercarse a su realidad operacional y administrativa, conocer su estado actual frente a la salud y seguridad en el trabajo y su articulación con la gestión de la calidad. Dentro de las actividades realizadas como parte de ese reconocimiento se adelantaron grupos focales para conocer las expectativas y conocimientos previos de los colaboradores alrededor de los temas mencionados, y a su vez para generar un proceso de redireccionamiento estratégico que contara con la participación del personal administrativo y operativo.

Para formular las políticas y los objetivos del SG-SST se utilizó la metodología gamestorming, una guía metodológica válida para la implementación de un sistema de gestión de seguridad y salud en el trabajo en pymes de Colombia cuya base es el libro 2, parte 2, titulo 4, capítulo 6 del Decreto 1072 de 2015. La implementación incluyó una serie de actividades didácticas tendientes a lograr la gestión del sistema y el compromiso de los colaboradores de la organización (Guarín-Montenegro 
y Varela Alonso, 2015). En total se realizaron cuatro actividades basadas en los parámetros de la metodología y desarrolladas mediante un grupo focal: 1) ronda de preguntas y lluvia de ideas relacionadas con la cultura del servicio, 2) medición del nivel de conciencia y conocimiento respecto a la salud y la seguridad en el trabajo, 3 ) establecimiento de la política y los objetivos de la salud y la seguridad en el trabajo y 4) análisis del estado actual y prospectivo de la gestión del cambio y la del riesgo.

De forma paralela se desarrolló la mesa de trabajo, conformada por investigadores y asesores de la Facultad de Estadística de la Universidad Santo Tomás, con el objetivo de diseñar una encuesta que permitiera conocer el nivel de satisfacción y las necesidades de los clientes frente a los siguientes criterios: 1) claridad de la información acerca de las condiciones del servicio, 2) atención al cliente, 3) productos y 4) servicios.

Una vez obtenidos y procesados los resultados de la encuesta, estos fueron utilizados como insumo para el análisis de las capacidades internas, operacionales y administrativas de la organización. En cuanto a sus capacidades externas se analizó el perfil de oportunidades y amenazas del entorno a través de matrices de evaluación del riesgo (Serna-Gómez, 2014).

Los niveles de evaluación para capacidades internas y externas se evaluaron así:

- Capacidades internas

1: si el encuestado considera que el criterio es una gran debilidad de la organización.

2: si el encuestado considera que el criterio es una debilidad de la organización.

3: si el encuestado considera que el criterio es una fortaleza de la organización.

4: si el encuestado considera que el criterio es una gran fortaleza de la organización.
- Capacidades externas

1: si el encuestado considera que el criterio es una gran amenaza para la organización.

2: si el encuestado considera que el criterio es una amenaza para la organización.

3: si el encuestado considera que el criterio es una oportunidad para la organización.

4: si el encuestado considera que el criterio es una gran oportunidad para la organización.

Como última etapa del diagnóstico se desarrolló la redefinición de temas estratégicos, con el objetivo de hacer un ejercicio de construcción colectiva del direccionamiento entre los colaboradores, los fundadores e investigadores. Allíse definieron con claridad el propósito organizacional y la ruta estratégica (metas a alcanzar), alineados a la naturaleza de la organización. En total se realizaron cuatro actividades basadas en los parámetros suministrados por el profesor Serna-Gómez: 1) redefinición de la misión, 2) redefinición de la visión, 3) redefinición de los objetivos estratégicos y 4) construcción de un organigrama.

\section{Metodología de inteǵración}

Con el objetivo de identificar los requisitos para la integración de los sistemas de gestión y diseñar la metodología se realizó una revisión documental que incluyó normas, guías, artículos de investigación y revistas especializadas. La búsqueda de información se centró en sistemas integrados de gestión y asuntos de estrategia en pequeñas y medianas empresas.

De acuerdo con la información recopilada, y con el objetivo de evitar la duplicidad en la información, se elaboró una matriz de comparación entre la guía PAS 99, la Estructura de Alto Nivel y Guía para la Integración de Sistemas de Gestión y la norma UNE 66177. Las variables de análisis utilizadas en la matriz fueron: año de la última actualización, alcance, metodología y vocabulario. 
Producto de la comparación realizada se determinó que la guía PAS 99 y la norma UNE 66177 solo se enfocan en la definición de requisitos, mientras que la Estructura de Alto Nivel va más allá: contribuye con la estrategia de las organizaciones ya que "facilita la gestión integrada de la calidad y la seguridad y salud en el trabajo y optimiza los recursos para el logro de los objetivos organizacionales" (Rodríguez-Rojas y Pedraza-Nájar, 2017, p. 68), además de ser una metodología que apropia buenas prácticas organizacionales.

Por otra parte, a partir de la revisión de artículos y revistas especializadas se identificaron los siguientes elementos importantes para la integración de sistemas de gestión, que fueron tenidos en cuenta para el diseño de la metodología: responsabilidad de la alta dirección, instrucciones de trabajo claras, comunicación y rendición de cuentas.

Kaplan y Norton (2014) describen el mapa estratégico como una herramienta que aporta a la competitividad de las organizaciones que lo implementan pues traduce las estrategias en resultados operacionales y administrativos desde las perspectivas de aprendizaje y crecimiento, interna, de los clientes y financiera, de acuerdo al cuadro de mando integral para realizar la integración de la gestión. Por eso se definieron los siguientes indicadores a partir del mapa de operacionalización de variables. Están basados en la definición ofrecida por Hernández, Fernández y Baptista, según la cual tales indicadores "describen el proceso lógico para construir un instrumento [:] transitar de la variable a sus dimensiones o componentes, luego a los indicadores y finalmente a los ítems o reactivos y sus categorías" (p. 211):

- Perspectiva de aprendizaje y crecimiento
3) Requerimientos de las partes interesadas.

4) Clima de seguridad.

5) Compromiso.

6) Bienestar y desarrollo.

7) Mejores prácticas organizacionales.

- Perspectiva interna

1) Crecimiento organizacional.

2) Motivación del personal.

3) Cambio organizacional.

4) Propósito compartido.

5) Procesos.

6) Actividades.

7) Comunicación.

8) Nivel de rol.

9) Nivel de redistribución percibida.

- Perspectiva partes interesadas

1) Control a la operación y la gestión.

2) Trazabilidad de controles operacionales.

3) Capacidad aplicada del colaborador.

4) Calidad de vida.

5) Productividad.

- Perspectiva de rentabilidad

1) Aprendizaje organizacional.

2) Organización como sistema.

3) Cultura organizacional.

La metodología de integración construida a través de los indicadores mencionados permitió obtener elementos comunes de cada una de las normas técnicas y modelos normativos, además de relacionar el mapa estratégico con el ciclo PHVA (figura 1).

1) Plan estratégico.

2) Liderazgo de la alta dirección. 
Fig̉ura 1. Descripción propuesta metodológica

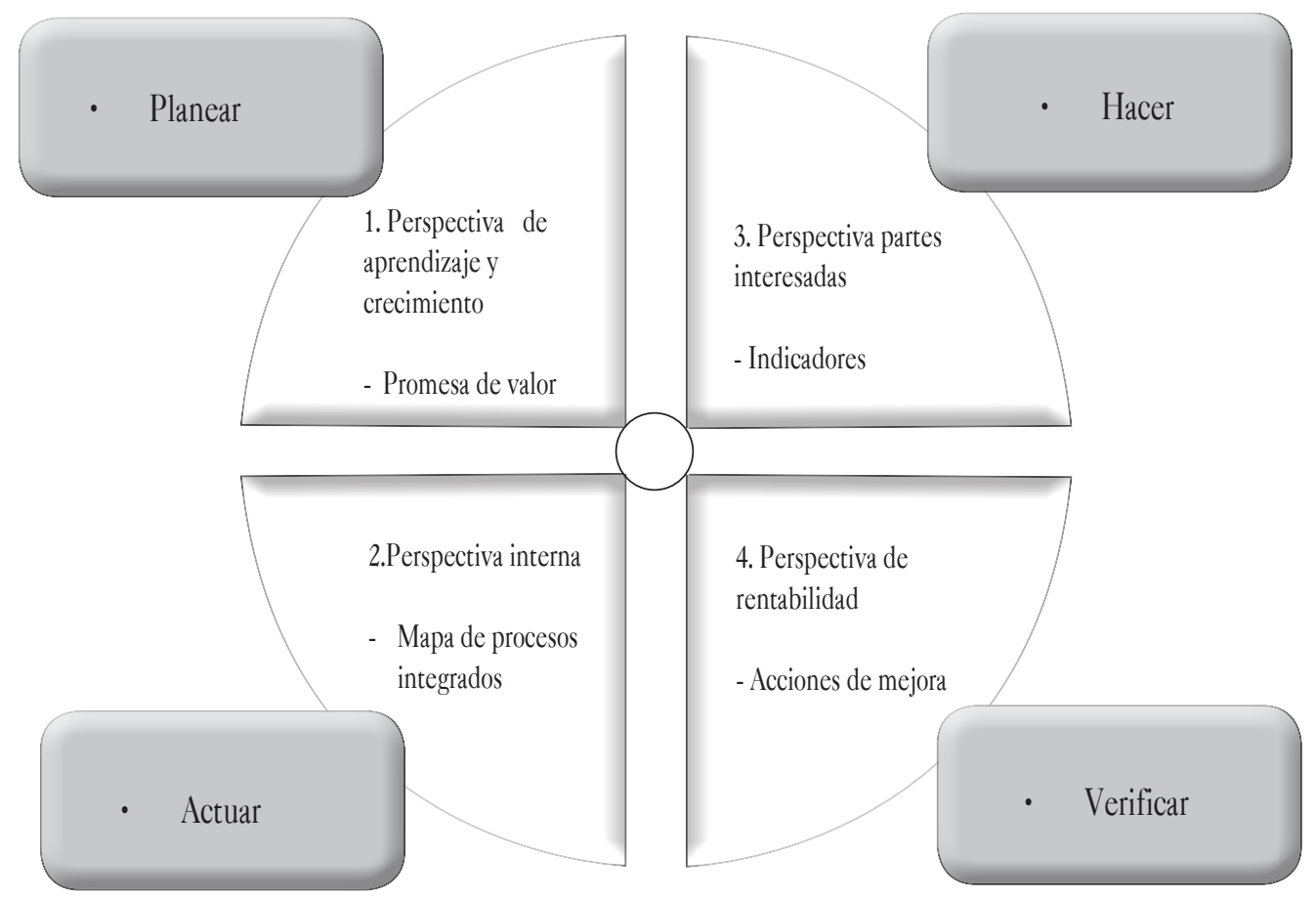

Fuente: elaboración propia.

\section{Validación de la metodología}

Finalizada la construcción de la metodología, se determina el proceso de recolección de datos cuantitativos para obtener criterios de validez definidos por Hernández et al. "como el grado en que un instrumento mide realmente la variable que pretende medir" (2014, p. 200). Desde ese punto de vista, en el estudio de caso se establecen dos criterios de validez: el primero, la pertinencia y relevancia, obtenidas a través de la validez de contenido del modelo propuesto por Lawshe (1975) y modificado por Tristán (2008) citado por Vargas, Guaderrama, Cavazos y Cervantes (2016, p. 35) ; el segundo, la coherencia o consistencia interna del instrumento, medida por medio del Alpha de Cronbach (Hungler y Polit, 2003).

En este contexto se desarrolló la aplicación de la metodología en la organización, lo que permitió reconocer y realimentar la propuesta por medio del diseño de investigación-acción. La labor se dividió en tres etapas repetitivas, a saber: observar (construir un bosquejo del problema y recolectar datos), pensar (analizar e interpretar) y actuar (resolver problemáticas e implementar mejoras), "y otra vez, hasta que todo es resuelto, el cambio se logra o la mejora se introduce satisfactoriamente" (Hernández et al., 2014, p. 497).

Para finalizar, se realizó un ejercicio de triangulación. Autores como Hungler y Polit la definen como "el uso de referentes múltiples para llegar a conclusiones acerca de lo que constituye la verdad y es usada para incrementar la probabilidad de que los resultados de estudios cualitativos sean creibles" (2003, p. 409). En nuestro estudio de caso la triangulación se desarrolló entre expertos (en sistemas de gestión e integrados), observadores 
Erika Rocío Rodríguez Rodríguez, Carlos Enrique Montenegro Narváez, Edgar Mauricio Mendoza García

(investigadores) y lideres de proyecto (fundadores y colaboradores), que entregaron datos individuales y colectivos con el objetivo de realimentar la propuesta.

\section{RESULTADOS Y DISCUSIÓN}

Una vez realizada la triangulación entre los conceptos y opiniones emitidos por los expertos, los colaboradores y el grupo de investigadores procesaron y analizaron los resultados relacionados con la aplicación y validación de la metodología de integración con el propósito de realimentar la propuesta.

\section{Resultados obtenidos}

En cuanto al diagnóstico sobre el estado actual de la salud y la seguridad en el trabajo en la organización analizada, las actividades desarrolladas (grupo focal), dirigidas a la alta dirección y los colaboradores, concluyeron con la elaboración de la política del SG-SST y los objetivos, así:
- Política

La organización se compromete a brindar las condiciones necesarias y oportunas para que los colaboradores desarrollen sus actividades en un buen ambiente, lo que ha de reflejarse en un excelente servicio a sus clientes y partes interesadas.

- Objetivo general

Conformar a mediano plazo un completo sistema de seguridad y salud en la organización, apoyado en todas las partes interesadas.

- Objetivos específicos

- Garantizar las herramientas necesarias para el desarrollo de las actividades laborales.

- Identificar peligros y riesgos dentro de la organización y realizar la debida gestión.

- Garantizar el cumplimiento de las normas establecidas por ambas partes (gerentes-colaboradores).

Figura 2. Matriz de riesgo satisfacción del negocio

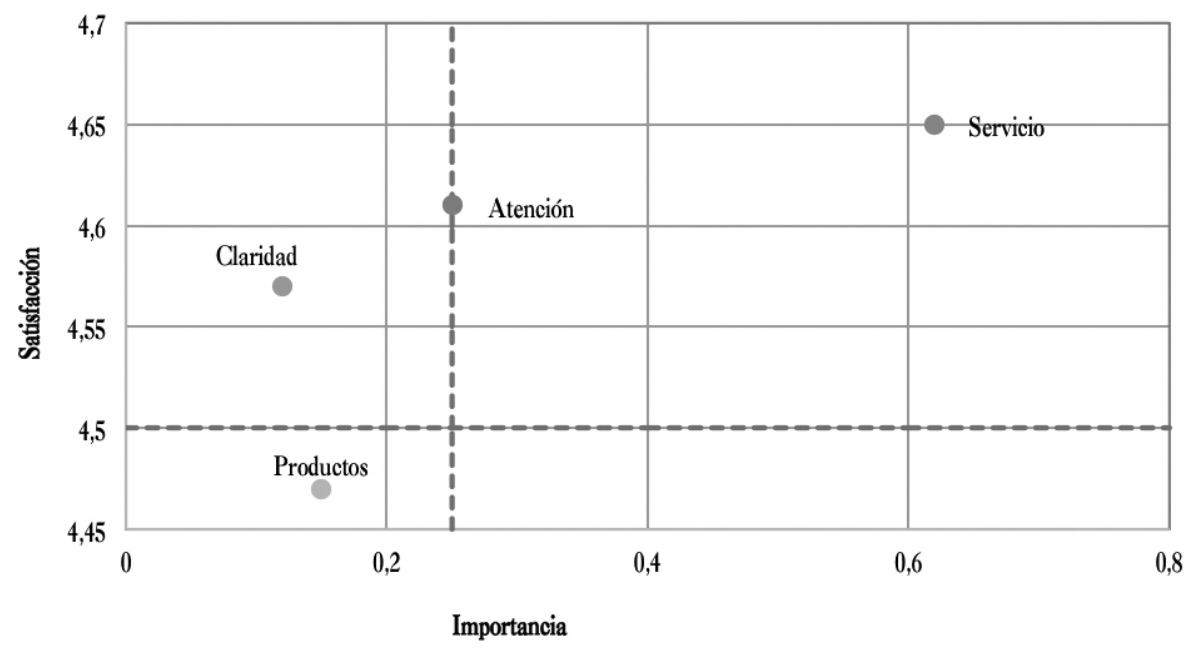

Fuente: elaboración propia. 
Una vez identificados los elementos principales del SG-SST se desarrolló la articulación con la gestión de la calidad por medio de una encuesta que permitió conocer el nivel de satisfacción y las necesidades de los clientes, la cual concluyó con nubes de palabras y una matriz de riesgo (figura 2) usadas para identificar fortalezas, debilidades y aspectos a mejorar.

En la matriz de riesgo de satisfacción general se puede observar que el ítem mejor calificado y de mayor importancia para los clientes es el de Servicios, que comprende los servicios de cerrajería, plomería y electricidad, con un resultado de importancia de 0.62 y uno de satisfacción de 4.65, este último obtenido principalmente por la capacidad de los colaboradores al momento de prestar los servicios. El segundo mejor calificado, con una importancia media (0.25), es el de Atención al cliente, con un resultado de satisfacción de 4.61. Por otro lado, los ítems con menor calificación fueron el de Productos y el de Claridad de la información.

Respecto a las condiciones iníciales de la organización frente a la gestión de la calidad, la salud y la seguridad en el trabajo, la encuesta aplicada a la alta dirección y los colaboradores mostró que se deben generar estrategias de crecimiento y desarrollo para mantener su posición en el medio, facilitar la consecución de una mayor cuota del mercado y mejorar su relación con las partes interesadas. La encuesta mostró en promedio, valores de 3.0 y 2.5 en la escala Likert definida para el contexto interno y el contexto externo, respectivamente (tabla 1).

De acuerdo con los resultados del diagnóstico organizacional se identificaron las principales fortalezas, oportunidades, debilidades y amenazas con el propósito de establecer las estrategias que guiarán el futuro de la organización.
Tabla 1. Análisis cuantitativo contexto interno y externo de la organización

\begin{tabular}{|l|c|c|}
\hline \multicolumn{3}{|c|}{ Contexto interno } \\
\hline Área & Importancia & Total \\
\hline Directivo & $15,00 \%$ & 0,53 \\
\hline Competitivo & $10,20 \%$ & 0,31 \\
\hline Financiero & $12,00 \%$ & 0,40 \\
\hline Tecnológico & $10,20 \%$ & 0,26 \\
\hline Talento Humano & $10,20 \%$ & 0,25 \\
\hline Logístico & $10,20 \%$ & 0,31 \\
\hline Operativo & $10,20 \%$ & 0,31 \\
\hline Marketing & $10,20 \%$ & 0,25 \\
\hline Ventas & $12,00 \%$ & 0,36 \\
\hline Total & $100 \%$ & 3.0 \\
\hline
\end{tabular}

\begin{tabular}{|l|c|c|}
\hline \multicolumn{3}{|c|}{ Contexto externo } \\
\hline Área & Importancia & Total \\
\hline Demográfico & $16,00 \%$ & 0,56 \\
\hline Económico & $24,90 \%$ & 0,59 \\
\hline Político/Legal & $25,00 \%$ & 0,47 \\
\hline Sociocultural & $10,00 \%$ & 0,25 \\
\hline Tecnológico & $16,00 \%$ & 0,46 \\
\hline Recursos Naturales & $8,00 \%$ & 0,16 \\
\hline Total & $100 \%$ & 2.5 \\
\hline
\end{tabular}

Fuente: elaboración propia.

- Fortalezas

- Direccionamiento estratégico: misión, visión y objetivos estratégicos claros.

- Diferenciación con la competencia: la organización cuenta con amplio inventario y rotación de productos.

- Interés por conocer las necesidades y expectativas de los clientes. 
- Oportunidades

- Crecimiento de la economía del país/crecimiento del sector construcción.

- El sector ferretero se está adaptando a necesidades y expectativas del mercado comercializando productos amigables con el medio ambiente.

- Debilidades

- Administración inadecuada de los recursos.

- Difícil adaptación al cambio.

- Amenazas

- Poder de negociación de las grandes superficies.

- Incremento de competencia externa (llegada de grandes ferreterías).

Finalmente, se propusieron las estrategias adecuadas al contexto de la organización, necesarias como insumo para la metodología de integración debido a que describen factores claves de éxito que generan valor. De acuerdo a lo anterior y con el objetivo de definir la ruta estratégica (metas a alcanzar) se realizó la redefinición de los temas estratégicos.

Al finalizar el diagnóstico organizacional se estableció un direccionamiento estratégico para dar inicio a un proceso de diferenciación a través de la construcción de la metodología de integración alineada con la estructura de alto nivel propuesta por la ISO. La metodología se desarrolló desde la definición de los ítems, como se muestra en la tabla 2.

En rigor, el SGI está orientado internamente por las necesidades y motivaciones personales del empresario, las cuales deben estar vinculadas con las necesidades y los objetivos organizacionales para equilibrar los beneficios que se desea obtener. Por otro lado, el SGI está orientado externamente por las demandas de los clientes, que a su vez están motivados por la propuesta de valor ofrecida por la organización.

Tabla 2. Operacionalización de variables-construcción de la metodología

\begin{tabular}{|l|l|}
\hline \multicolumn{1}{|c|}{ Codificación } & \multicolumn{1}{c|}{ Ítems } \\
\hline Ítem 01 & $\begin{array}{l}\text { La organización cuenta con un plan estratégico para enfocarse en las actividades que generan valor a sus } \\
\text { clientes y contribuir a la definición de una propuesta de valor }\end{array}$ \\
\hline Ítem 02 & $\begin{array}{l}\text { El sistema integrado de gestión es un reflejo de las intenciones de los líderes de generar rentabilidad, } \\
\text { fortalecer los procesos internos y desarrollar conciencia de mejores prácticas }\end{array}$ \\
\hline Ítem 03 & $\begin{array}{l}\text { El sistema de gestión integrado propone un equilibrio entre las necesidades del empresario y las necesidades } \\
\text { y objetivos organizacionales para el logro del crecimiento y el aprendizaje organizacional }\end{array}$ \\
\hline Ítem 04 & $\begin{array}{l}\text { Los requisitos de ley han promovido el cambio y la adaptación proactiva para implementar el sistema de } \\
\text { gestión integrado }\end{array}$ \\
\hline Ítem 05 & $\begin{array}{l}\text { El sistema de gestión integrado considera las necesidades y expectativas de las partes interesadas para la } \\
\text { generación de una cultura de servicio }\end{array}$ \\
\hline Ítem 06 & $\begin{array}{l}\text { El sistema de gestión integrado es promovido por una necesidad de cambio estructural para contribuir al } \\
\text { crecimiento organizacional }\end{array}$ \\
\hline Ítem 07 & $\begin{array}{l}\text { La identificación de las necesidades y expectativas de las partes interesadas genera un propósito compartido } \\
\text { que incentiva la participación, el compromiso y la cooperación, lo que contribuye a la sostenibilidad }\end{array}$ \\
\hline
\end{tabular}




\begin{tabular}{|c|c|}
\hline Codificación & Ítems \\
\hline Ítem 08 & $\begin{array}{l}\text { El mapa de procesos permite integrar los procesos claves del SGC (sistema de gestión de calidad) y el SG- } \\
\text { SST (sistema de seguridad y salud en el trabajo), lo que permite la optimización de recursos }\end{array}$ \\
\hline Ítem 09 & $\begin{array}{l}\text { La identificación de los procesos clave permite visualizar qué actividades pueden generar el incumplimiento } \\
\text { de las características de calidad y ocasionar peligros a la organización }\end{array}$ \\
\hline Ítem 10 & $\begin{array}{l}\text { El sistema de gestión integrado incrementa el grado de interacción entre las estructuras funcionales, lo que } \\
\text { considera la necesidad de que exista mayor comunicación y cooperación entre ellas }\end{array}$ \\
\hline Ítem 11 & $\begin{array}{l}\text { Establecer los controles operacionales adecuados de las actividades internas orienta la operación y gestión de } \\
\text { la organización }\end{array}$ \\
\hline Ítem 12 & $\begin{array}{l}\text { De acuerdo a la definición de los controles operacionales se realiza el análisis de trazabilidad para verificar la } \\
\text { eficacia de las responsabilidades asignadas al sistema de gestión integrado }\end{array}$ \\
\hline Ítem 13 & $\begin{array}{l}\text { El aprendizaje en los distintos niveles de la organización permite que las personas se adapten al cambio y } \\
\text { contribuyan a los propósitos organizacionales }\end{array}$ \\
\hline Ítem 14 & $\begin{array}{l}\text { Una de las ventajas del sistema de gestión integrado es aumentar la competitividad de las organizaciones } \\
\text { mediante la adopción de mejores prácticas que se reflejan en el producto o servicio que ofrece la organización } \\
\text { en respuesta a las necesidades del cliente }\end{array}$ \\
\hline Ítem 15 & $\begin{array}{l}\text { La organización diseña el sistema de gestión integrado contemplando a las personas, sus motivaciones e } \\
\text { intereses, sus competencias y potencialidades con el propósito de lograr mejores condiciones laborales }\end{array}$ \\
\hline Ítem 16 & $\begin{array}{l}\text { El éxito del sistema de gestión integrado está relacionado con las motivaciones que llevan a la organización a } \\
\text { avanzar hacia la mejora de la imagen corporativa }\end{array}$ \\
\hline Ítem 17 & $\begin{array}{l}\text { El sistema de gestión integrado permite mejorar las condiciones y los ambientes en la organización, lo que } \\
\text { facilita el bienestar y desarrollo de los colaboradores }\end{array}$ \\
\hline Ítem 18 & $\begin{array}{l}\text { Motivar comportamientos y comunicar una visión orientada a la seguridad impacta en mejores prácticas } \\
\text { organizacionales }\end{array}$ \\
\hline Ítem 19 & $\begin{array}{l}\text { Al determinar e incorporar las habilidades individuales de desarrollo de los colaboradores el sistema de gestión } \\
\text { integrado promueve las condiciones de calidad y seguridad en la organización }\end{array}$ \\
\hline Ítem 20 & $\begin{array}{l}\text { El sistema de gestión integrado reconoce el aporte individual de los colaboradores a través del apoyo recibido } \\
\text { por el trabajo realizado }\end{array}$ \\
\hline Ítem 21 & $\begin{array}{l}\text { El sistema de gestión integrado tiene en cuenta la capacidad aplicada del colaborador, reflejada en el } \\
\text { cumplimiento de las tareas y los objetivos delegados por el líder de la organización }\end{array}$ \\
\hline Ítem 22 & $\begin{array}{l}\text { El sistema integrado de gestión permite que la organización logre sus objetivos en beneficio de todos, de } \\
\text { modo que obtengan un mejor nivel en su calidad de vida }\end{array}$ \\
\hline Ítem 23 & $\begin{array}{l}\text { El sistema integrado de gestión permite que la organización logre sus objetivos en beneficio de todos, lo que } \\
\text { facilita el desarrollo personal de sus miembros }\end{array}$ \\
\hline Ítem 24 & $\begin{array}{l}\text { Una organización competitiva es el resultado del aprendizaje y la adaptación al entorno, que permite a los } \\
\text { colaboradores adquirir mejores prácticas de cuidado y bienestar en el lugar de trabajo }\end{array}$ \\
\hline
\end{tabular}

Fuente: elaboración propia. 


\section{La integración desde la óptica de la realidad de las organizaciones}

La realidad de las organizaciones es que están integradas al contexto global debido a que el desarrollo de sus actividades se ve afectado por ese contexto. El reto está en poder integrar la gestión desde la planificación y disminuir el impacto generado en el desarrollo diario de las actividades.

Una organización compleja es aquella que por su contexto no se desarrolla rápidamente en términos de tecnología, procesos y recursos humanos. Sin duda, una organización mipyme tiene facilidades para coordinar sus procesos y comunicarlos debido al tamaño de su estructura y la cantidad de colaboradores que contiene, pero hay que tener en cuenta que el empresario mipyme realiza la mayoría de sus actividades pensando en el día a día. Por supuesto, no es objetivo de la metodología seguir cultivando un mal hábito; por el contrario, el SGI (sistema de gestión integrado) busca transformar la cultura organizacional, lo que implica el cambio de estilo de dirección del líder de la alta dirección y su influencia en los colaboradores. Por lo tanto, trabajar en la estrategia o en la integración se convierte en un reto que necesita del apoyo del recurso humano, disponibilidad de tiempo y conocimientos previos sobre la organización y el sector.

En rigor, el SGI está orientado internamente por las necesidades y motivaciones personales del empresario, las cuales deben estar vinculadas con las necesidades y los objetivos organizacionales para equilibrar los beneficios que se desea obtener. Por otro lado, el SGI está orientado externamente por las demandas de los clientes, que a su vez están motivados por la propuesta de valor ofrecida por la organización. Se debe considerar la importancia de los agentes facilitadores como generadores de cambios que aportan a la adaptación proactiva del SGI. La organización pyme tiene la facilidad de ofrecer un tratamiento individualizado a las partes interesadas y por eso tiene la oportunidad de conocer sus gustos, preferencias e inconformidades. Por lo tanto, se construye una cultura de servicio para facilitar la integración involucrando a los colaboradores como causantes de una buena atención.

Por lo anterior, la metodología de integración contempla las competencias y potencialidades de cada colaborador con el propósito de lograr mejores condiciones laborales para facilitar el bienestar y desarrollo de las personas. Ello se consolida beneficiando al agente facilitador sistema empresarial con mejores prácticas al implementar el SGC y el SG-SST, lo que permite a su vez avanzar hacia la mejora de la imagen corporativa.

\section{La integración desde la planificación de las especificaciones}

Se dice que los sistemas de gestión tienen funciones diferentes y no se pueden integrar. Como consecuencia, la integración se desarrolla en términos de la gestión, partiendo de la estructura de alto nivel, que considera el ciclo PHVA, para lograr la eficacia y eficiencia del sistema a través de un enfoque por procesos 2 .

Para llevar a cabo la integración se debe tener en cuenta el enfoque de cada sistema: el de calidad (SGC) está dirigido al cliente; el de seguridad y salud en el trabajo (SG-SST), a las personas de la organización.

De acuerdo con lo planteado anteriormente, la organización debe reconocer las entradas, actividades y salidas de cada proceso que involucra a los sistemas para identificar las características de calidad y los peligros a los que se ven expuestos estos por el desarrollo de las actividades. A esto se añade la definición de las especificaciones de cada sistema traducidas como la promesa de

2 Peña. G. (2018). Seminario Gestión Integral, Maestria en Calidad y Gestión Integral Usta-Icontec, Bogotá. 
valor de acuerdo a las capacidades de la organización. A partir de las especificaciones se empieza a construir una relación de compromiso con las partes interesadas que garantiza la sostenibilidad de la organización.

La estructura de la metodología parte de la perspectiva de crecimiento y aprendizaje, en la que se planifican las especificaciones identificadas como la promesa de valor, que son definidas para dar cumplimiento a las expectativas de las partes interesadas.

En la perspectiva interna se desarrolla el mapa de procesos identificándolos así: procesos misionales, directamente asociados al propósito organizacional; procesos de apoyo, que suministran los recursos necesarios para que la integración funcione, y procesos estratégicos, requeridos para el cumplimiento de metas. El mapa de procesos integrado tiene como entrada las necesidades de las partes interesadas (especificaciones), implementadas en las diferentes actividades de cada proceso, y la salida o efecto es el producto o servicio ya terminado.

Por supuesto, para que la organización pueda hacer la verificación y validación del cumplimiento de las promesas de valor a las partes interesadas es necesario identificar los actores involucrados en el seguimiento. En el caso de la metodología, como está enfocada en la ISO 9001:2015 y el SG-SST sus respectivos actores son el cliente y los colaboradores. Por lo tanto, es fundamental realizar el seguimiento a las quejas y los reclamos.

Por último, se desarrolla la perspectiva de rentabilidad, que considera los activos tangibles materializados dentro de las acciones de mejora pertinentes para el sistema integrado de gestión. Muestra los resultados de los indicadores de cada perspectiva.

A partir del diseño de la metodología y con los datos recopilados se realizó el tratamiento estadístico (tabla 3). El primer criterio fue el IVC (índice de validez de contenido), que se evaluó mediante las ecuaciones de Lawshe con factor de corrección de Tristan, que da como resultado valores de pertinencia y relevancia de 0.66 a 1 . De esta forma, obtener calificaciones a partir de 0.66 implica que los seis expertos aceptaron cada ítem al proporcionar calificaciones de 304 en pertinencia y relevancia.

El segundo criterio de aceptación fue el cálculo del alfa de Cronbach. El resultado fue de 0.9886, por encima del valor de aceptación (de 0.8 ), lo que confirmó la conclusión aportada por el cálculo del IVC de pertinencia y relevancia sobre la fiabilidad de la metodología de integración recomendada para la organización.

Por último, al aplicar la metodología de integración se propuso la alineación estratégica para la organización con base en un árbol (figura 3). Las raíces son las perspectivas de aprendizaje y crecimiento, los procesos internos, los clientes y el factor financiero cada uno interactuando para generar valor a la organización. El direccionamiento estratégico es el tronco del árbol, entendido como el soporte organizacional donde se sitúan las estrategias adecuadas al contexto y del que nacen las ramas del SGI, que garantizarán la sostenibilidad ayudando a cumplir los objetivos estratégicos y previniendo accidentes para no afectar la rentabilidad. 
Tabla 3. Resultados validación de contenido por panel de expertos

\begin{tabular}{|c|c|c|c|}
\hline \multicolumn{4}{|c|}{$\begin{array}{l}\text { Resultados: IVC (índice de validez de contenido) y coeficiente de alfa de Cronbach } \\
\text { Criterio de aceptación: CVI > } 0.8 \text { y } \alpha>0.8\end{array}$} \\
\hline Ítems & $\begin{array}{c}\text { CVI } \\
\text { Pertinencia }\end{array}$ & CVI Relevancia & Alfa de Cronbach \\
\hline 24 & 0.8542 & 0.9167 & 0.9886 \\
\hline
\end{tabular}

Fuente: elaboración propia.

Figura 3. Árbol de crecimiento y sostenibilidad

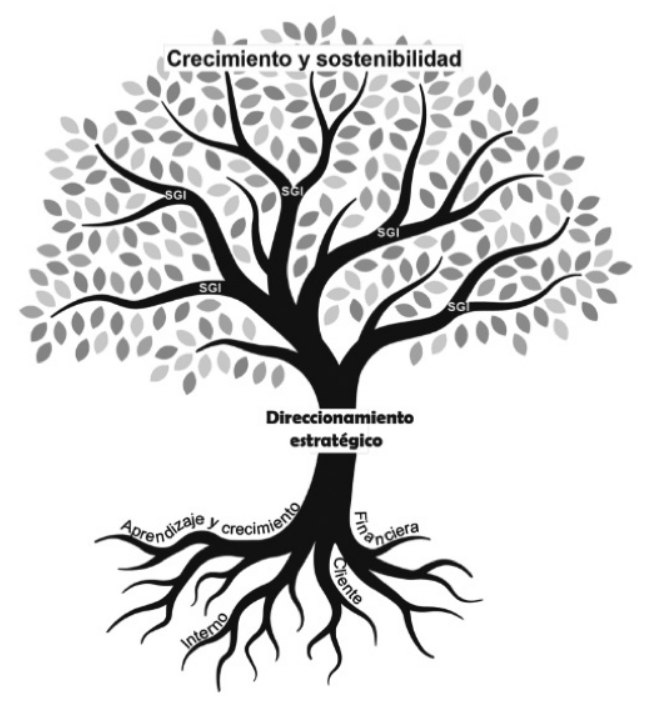

Fuente: elaboración propia

\section{CONCLUSIONES}

¿Cómo desarrollar una metodología para la integración de la gestión en una mipyme del sector comercio a partir de la ISO 9001:2015 y el Decreto 1072 de 2015, que influya su crecimiento y sostenibilidad? Como respuesta se presentó esta propuesta metodológica a partir del direccionamiento estratégico de la organización, resultado final del proceso de investigación descrito.
En primer lugar, se establece que el análisis interno de la organización obtuvo un promedio de 3 y el análisis externo un promedio de 2.5 en la escala tipo Likert, lo cual corrobora que la organización de acuerdo a su situación actual debe buscar estrategias para crecer y desarrollarse. Los objetivos son mantenerse, ver la posibilidad de obtener una mayor cuota de mercado, potencializar los productos y servicios ofrecidos, atraer y retener nuevos clientes y ser reconocida por la prestación de un buen servicio, la disposición, la variedad de productos, la calidad de los artículos y un entorno de trabajo seguro.

En segundo lugar, se llevó a cabo la construcción del mapa de operacionalización de variables. Teniendo como base los elementos proporcionados por los autores citados en el marco teórico se establecieron veinticuatro items (afirmaciones), los cuales permitieron definir los lineamientos del diseño de la metodología para la integración de la gestión.

Una vez obtenida la metodología de integración construida a partir de los ítems de la operacionalización de variables se consideró la aplicación de un panel de expertos, siguiendo a Hungler y Polit (2003), para evaluar pertinencia y relevancia. Asimismo, se seleccionó el método estadístico para evaluar validez de contenido a partir de las ecuaciones de Lawshe con el factor de corrección propuesto por Tristán-López, que permite 
la aplicación del panel a partir de cinco expertos, adecuándose a la investigación en cuestión. Los expertos analizaron la metodología propuesta y respondieron el instrumento de validación.

Posteriormente, al realizar la validación de contenido por medio del panel de expertos, se confirmó que la metodología permite la integración de la gestión, al obtener resultados aprobatorios en los criterios de validez de contenido tanto en términos de pertinencia $(\mathrm{IVC}=0.8542)$ como de relevancia $(\mathrm{IVC}=0.9167)$. Igualmente, el resultado del cálculo del alfa de Cronbach $(\alpha=0.9886)$ corrobora la confiabilidad de los veinticuatro ítems del instrumento de validación de contenido.

Es decir, la metodología diseñada permite la gestión integral para la organización bajo estudio (Electrocerraduras la 85), de acuerdo con los resultados de la validación de contenido por expertos. No obstante, se consideró significativo realizar la aplicación de la metodología diseñada a los integrantes de la organización en aras de reconocer y realimentar la propuesta. Este ejercicio permitió establecer los cambios pertinentes, la viabilidad del proyecto y su alineación estratégica. Teniendo en cuenta lo anterior, los resultados fueron la construcción del mapa estratégico y el árbol de crecimiento y sostenibilidad de Electrocerraduras la 85, que en conjunto constituyen la planeación del SGI.

Por último, los resultados de la simulación en la organización demuestran la viabilidad de la metodología en lo financiero, lo táctico y lo operativo:

- Dimensión financiera: la simulación desarrolló el cuadro de mando integral, el cual identifica los indicadores organizacionales que impactarán los resultados financieros.
- Dimensión táctica: se identificaron los roles y responsabilidades de cada persona en la organización con respecto al SGI. Además, se impulsó la gestión del cambio que contribuye al crecimiento organizacional por medio de la toma de conciencia que se llevó a cabo en la fase I de la investigación (acercamiento organizacional).

- Dimensión operativa: se indicaron instrucciones de trabajo y se identificaron controles en los diferentes procesos misionales.

A partir de la recolección obtenida y la información aportada por expertos, colaboradores e investigadores se realiza la triangulación que permitió identificar puntos en común para obtener la metodología de integración realimentada.

Posteriormente se presentan las recomendaciones, orientadas a futuras investigaciones que permitan la continuación de la línea de investigación de los SGI para el crecimiento y sostenibilidad de las organizaciones mipyme en el país:

- La organización debe adaptarse a los cambios constantes de su entorno. Por lo tanto, debe realizar una vez al año revisión del direccionamiento estratégico.

- Incorporar a la metodología de integración nuevos constructos, tales como gestión del riesgo, innovación o responsabilidad social corporativa.

- Para la continuidad del proyecto es necesario realizar la verificación e impulsar la mejora continua, con el objetivo de pasar por todas las fases del ciclo PHVA.

- Se sugiere para la continuación del proyecto contar con una batería de instrumentos que indiquen cómo implementar los debes que establecen las normas. 


\section{REFERENCIAS}

Centro de Estudios Económicos (2018). La gran encuesta pyme. Bogotá: Anif.

Guarín-Montenegro G. y Varela-Alonso, C. (2015). Guía metodológica para la implementación del sistema de gestión de seguridady salud en el trabajo basada en gamestorming para mipymes en Colombia. Bogotá: Usta/Icontec.

Hernández, R., Fernández, C. y Batista. P. (2014). Metodología de la investigación. México: McGraw-Hill Education.

Hungler, B. P. y Polit, D. (2003). Evaluación de la calidad de los datos. En B. P. Hungler, Investigación científica en ciencias de la salud: principios y métodos. México: McGraw-Hill Interamericana.

Jørgensen, T., Remmen, A. y Mellado M. (2006). Integrated Management Systems: Three different levels of integration. Journal of Cleaner Production, 14(8), 713-722. Doi: https://doi.org/10.1016/j.jclepro.2005.04.005

Nunhes, T. V., Motta Barbosa, Luis César F, \& de Oliveira, O. J. (2017). Identification and analysis of the elements and functions integrable in integrated management systems doi://doi.org.bdatos.usantotomas.edu.co/10.1016/j.jclepro.2016.10.147

Portafolio (22 de agosto de 2016). Ferreterías: "con todos los fierros" para aprovechar diciembre. Recuperado de https://tinyurl.com/y6ekcsdk.
Rebelo, M., Santos, G. y Silva, R. (2016). Integration of management systems: Towards a sustained success and development of organizations. Journal of Cleaner Production, 127, 96-111.

Rodríguez-Rojas, Y. L. y Pedraza-Nájar, X. L. (2017). Aportes de la estructura de alto nivel en la gestión integrada. Revista Global de Negocios, 5 (2), 65-75.

Rodríguez-Rojas, Y. L. y Molano, J. H. (2018). Estrategias para la mejora de la gestión de la seguridad y salud en el trabajo frente a las formas de vinculación en un consorcio empresarial. Universidad Nacional de Colombia. EN: A.I. Muñoz. Salud de los colectivos. Reflexiones, metodologías y experiencias en salud y seguridad en el trabajo en Colombia. Bogotá, Colombia: Universidad Nacional de Colombia.

Salazar Castillo, José Manuel y Zarandona Azkuenaga, $X$. Análisis comparativo de las implicaciones organizativas de la calidad y de la gestión del conocimiento. En: El comportamiento de la empresa ante entornos dinámicos: XIX Congreso anual y XV Congreso Hispano Francés de AEDEM (Comunicaciones). p. 1-9.

Serna-Gómez, H. (2014). Gerencia estratégica. Bogotá: Panamericana.

Vargas, S., Guaderrama, M., Cavazos, A y Cervantes, B (2016). Validez de contenido de un instrumento de medición para medir el liderazgo transformacional. Revista Global de Negocios. p. 35-45. 